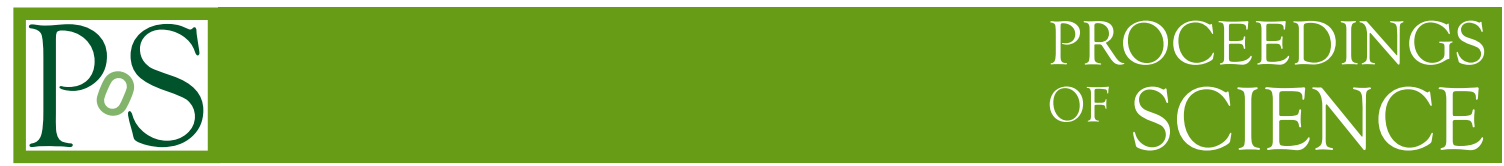

\title{
Properties of chiral magnetohydrodynamics
}

\section{Yuji Hirono*}

Brookhaven National Laboratory

E-mail: yhirono@bnl.gov

The chiral magnetic effect (CME) is a macroscopic manifestation of the quantum anomaly. There has been rapid progress in theoretical understanding the nature of chiral plasmas, in which the CME and other anomaly-induced transports take place. In this talk, we discuss the properties of the chiral magnetohydrodynamics (MHD), which is a hydrodynamic theory of chiral plasmas with dynamical electromagnetic fields. We introduce the formulation of the theory, and discuss linear excitations.

Critical Point and Onset of Deconfinement - CPOD2017

7-11 August, 2017

The Wang Center, Stony Brook University, Stony Brook, NY

${ }^{*}$ Speaker. 


\section{Introduction}

There have been growing interests in the physics of chiral materials. Remarkably, the chiral anomaly, which is a quantum effect, results in macroscopic non-dissipative transport effects. Those effects can be summarized in the following constitutive relations for the vector and axial currents,

$$
\begin{aligned}
& j_{\mathrm{V}}^{\mu}=\xi_{\mathrm{B}} B^{\mu}+\xi_{\omega} \omega^{\mu}, \\
& j_{\mathrm{A}}^{\mu}=\zeta_{\mathrm{B}} B^{\mu}+\zeta_{\omega} \omega^{\mu},
\end{aligned}
$$

where $B^{\mu} \equiv \tilde{F}^{\mu v} u_{v}$ with $\tilde{F}_{\mu v} \equiv \frac{1}{2} \varepsilon_{\mu v \alpha \beta} F^{\alpha \beta}, u^{\mu}$ is the fluid velocity, $\omega_{\mu} \equiv \frac{1}{2} \varepsilon_{\mu v \alpha \beta} u^{v} \partial^{\alpha} u^{\beta}$ is the vorticity tensor. The transport coefficients for the chiral magnetic effect (CME) $[1,2] \xi_{\mathrm{B}}$ and the chiral separation effect (CSE) $\zeta_{\mathrm{B}}$ are solely determined by the anomaly coefficient and they do not get further quantum corrections. The universal nature of the anomalous transport coefficients is checked by various calculation techniques such as the perturbation theory, lattice QCD $[3,4]$, the kinetic theory [5, 6, 7], and the hydrodynamics [8]. The derivation of those effects in terms of hydrodynamics [8] exemplifies the fact that they are macroscopic transport effects. There have also been experimental efforts to realize those effects as well as theoretical investigations. The CME is recently detected in Dirac semimetals [9]. There has been on-going vigorous efforts in heavy-ion collisions experiments to find the evidence of such effects, too.

In the present talk, we will discuss the recent developments in understanding the anomalous transport effects. Our focus here is particularly on the effects of dynamical electromagnetic fields. In most cases, the anomalous transport effects including the CME are discussed as a response to applied fields.

In reality, the generated currents will backreact to the electromagnetic fields and modify them. The fields and currents have to be treated as a coupled system, and we need a consistent framework describe such a system. We call the low-energy effective theory for this purpose as the chiral magnetohydrodynamics (MHD). The chiral MHD can be formulated as a derivative expansion, just as in the ordinary hydrodynamics. Together with the constraint from the second law of thermodynamics, the constitutive equations for the chiral MHD are derived, that include the chiral magnetic current.

\section{Topology of the magnetic fields and the cChiral anomaly}

Before delving into the chiral MHD, let us remind you an interesting relation as to the chiral anomaly and the topological properties of magnetic fields. The chiral anomaly is represented by the following equation,

$$
\partial_{\mu} j_{\mathrm{A}}^{\mu}=C_{\mathrm{A}} E \cdot B
$$

If we perform the spatial integration of Eq. (2.1), we obtain

$$
\frac{d}{d t}\left[\mathscr{H}+\mathscr{H}_{F}\right]=0
$$

where we have defined two helicities,

$$
\mathscr{H} \equiv \int d^{3} x \boldsymbol{A} \cdot \boldsymbol{B}, \quad \mathscr{H}_{F} \equiv \frac{2}{C_{\mathrm{A}}} \int d^{3} x n_{A} .
$$


We call the former one as the magnetic helicity, and the latter one as the fermionic helicity. Equation (2.2) says that the sum of two helicities are constant in time, although each of them are not necessarily conserved. It is well known that the magnetic helicity is a quantity that has the information of the topology of the field configuration. We can write the magnetic helicity with topological invariants as

$$
\mathscr{H}=\sum_{i} \mathscr{S}_{i} \varphi_{i}^{2}+2 \sum_{i, j} \mathscr{L}_{i j} \varphi_{i} \varphi_{j}
$$

when the field consists of magnetic flux tubes. Here, $\varphi_{i}$ represents the magnetic flux of the $i-$ th flux tube, $\mathscr{L}_{i j}$ is the Gauss linking number $\mathscr{S}_{i}$ is the Călugăreanu self-linking number. [10, 11, 12]. $\mathscr{L}_{i j}$ and $\mathscr{S}_{i}$ are topological invariants and they do not change values under continuous deformations of the configuration. The fact that the two helicities can be converted to each other means that, the presence of massless fermions can change the topology of magnetic fields, which is not possible without them.

Indeed, one can derive a formula connecting the the topology change of magnetic fields and CME current [13],

$$
\sum_{i} \oint_{C_{i}} \Delta \boldsymbol{J} \cdot d \boldsymbol{x}=-\frac{e^{3}}{2 \pi^{2}} \Delta \mathscr{H}
$$

where $e$ is the electric charge, where $\Delta \boldsymbol{J}$ is the amount of generated CME currents, $C_{i}$ represent the center lines of the magnetic flux tubes, and $\Delta \mathscr{H}$ is the change in magnetic helicity, From Eq. (2.5), we can conclude that, when the topology of the fields is modified, it has to result in the generation of CME currents. Such relation can be extended to include the topology of fluid flows [14]. If magnetic fields or vortex lines are reconnected, we automatically have CME currents, and they are quantified by Eq. (2.5) (see Fig. 2).

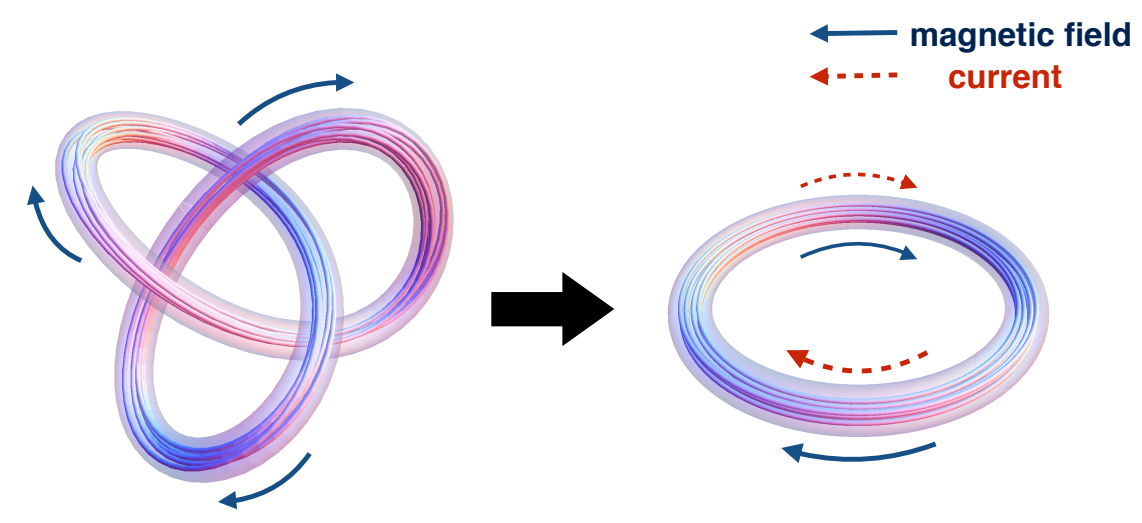

Figure 1: CME current generation due to the change of the topology of magnetic fields.

\section{Chiral MHD equations}

Hydrodynamics is a universal framework to describe systems at low energies. We study the long-time and long-wavelength dynamics of a chiral plasma, following the conventional logic of hydrodynamics [15]. We first consider locally equilibrium configurations. Those states are 
parametrized by Lagrange multipliers, $\left\{T, u^{\mu}, B^{\mu}, \mu_{\mathrm{A}}\right\}$, where $T$ is the temperature, $\mu_{\mathrm{A}}$ is the chiral chemical potential, $u^{\mu}$ is the fluid velocity.

Here, we did not treat the electric chemical potential $\mu$ as a hydrodynamic variable. The reason is that it is actually not a slow variable. Let us consider the conservation of the electric current,

$$
\partial_{t} n+\nabla \cdot \boldsymbol{j}=0 .
$$

If we use the Ohm's law $\boldsymbol{j}=\sigma \boldsymbol{E}$ the Gauss law $\nabla \cdot \boldsymbol{E}=n$, we obtain

$$
\partial_{t} n=-\sigma n .
$$

This equation indicates that $n$ is damping with the timescale determined by the electric conductivity $\sigma$. For this reason, $n$ is no longer a slow variable.

The equations governing the chiral MHD are given by the energy-momentum conservation, the Bianchi identity and the anomaly equation as

$$
\begin{aligned}
& \partial_{\mu} T^{\mu v}=0, \\
& \partial_{\mu} \tilde{F}^{\mu v}=0, \\
& \partial_{\mu} J_{\mathrm{A}}^{\mu}=C_{\mathrm{A}} E \cdot B,
\end{aligned}
$$

where $T^{\mu \nu}$ is the total energy-momentum tensor, and $C_{\mathrm{A}}=e^{2} /\left(4 \pi^{2}\right)$ is the anomaly coefficient. We shall express the quantities $\left\{T^{\mu \nu}, J_{\mathrm{A}}^{\mu}, \tilde{F}^{\mu \nu}\right\}$ by the hydrodynamic variables, order by order in the number of derivatives. The zeroth order terms define the ideal chiral MHD. At the zeroth order, the electric field vanishes, $E_{(0)}^{\mu}=0$. This means that the charge redistribution is fast. We formulate the chiral MHD as a double expansion in terms of derivatives and the anomaly coefficient.

\subsection{Zeroth order}

Let us discuss the zeroth order contributions in derivative and the anomaly coefficient. In general, the energy-momentum tensor, field-strength tensor and the axial current is written as

$$
\begin{aligned}
T_{(0)}^{\mu v} & =\varepsilon u^{\mu} u^{v}-X \Delta^{\mu v}-Y B^{\mu} B^{v}, \\
\tilde{F}_{(0)}^{\mu v} & =B^{\mu} u^{v}-B^{v} u^{\mu}, \\
J_{\mathrm{A}(0)}^{\mu} & =n_{\mathrm{A}} u^{\mu},
\end{aligned}
$$

where $\Delta^{\mu v} \equiv g^{\mu v}-u^{\mu} u^{v}$ is the spatial projector, and $\varepsilon$ is the energy density. The quantities $\varepsilon$, $X$, and $Y$ are functions of the hydrodynamic variables, $\left\{T, \mu_{\mathrm{A}}, B^{2}\right\}$. We here work in the Landau frame, and hence we do not have a term $\propto B^{\mu} u^{v}$ in $T^{\mu v}$. With the Eqs. (3.6),(3.7) and ((3.8)), the equations of motions is written as

$$
\begin{aligned}
u_{v} \partial_{\mu} T_{(0)}^{\mu v} & =\partial_{\tau} \varepsilon+\varepsilon \theta+X \theta-Y u_{v}(B \cdot \partial) B^{v}=0, \\
\partial_{\mu} \tilde{F}_{(0)}^{\mu v} & =\partial_{\tau} B^{\mu}+B^{\mu} \theta-(B \cdot \partial) u^{\mu}-u^{\mu}(\partial \cdot B)=0, \\
\partial_{\mu} J_{A(0)}^{\mu} & =\partial_{\tau} n_{\mathrm{A}}+n_{\mathrm{A}} \theta=0,
\end{aligned}
$$

where $\partial_{\tau} \equiv u_{\mu} \partial^{\mu}$ and $\theta \equiv \partial \cdot u$. The RHS of the anomaly equation is zero at this order. We use the thermodynamic relation,

$$
d \varepsilon=T d s+\mu_{\mathrm{A}} d n_{\mathrm{A}}+H_{\mu} d B^{\mu} .
$$


Let us rewrite this as

$$
d s=\beta d \varepsilon-\bar{\mu}_{\mathrm{A}} d n_{\mathrm{A}}-\beta H_{\mu} d B^{\mu},
$$

where we introduced $\beta \equiv 1 / T$ and $\bar{\mu}_{\mathrm{A}} \equiv \mu_{\mathrm{A}} / T$. Here $H^{\mu}$ is the magnetic field in medium.

We calculate the divergence of the entropy current, $S^{\mu}=s u^{\mu}$, which reads

$$
\begin{aligned}
\partial \cdot S= & \partial_{\tau} s+s \theta \\
= & \beta \partial_{\tau} \varepsilon-\bar{\mu}_{\mathrm{A}} \partial_{\tau} n_{\mathrm{A}}-\beta H_{\mu} \partial_{\tau} B^{\mu}+s \theta \\
= & \beta \theta\left(-X-\varepsilon+s T+\mu_{\mathrm{A}} n_{\mathrm{A}}+H \cdot B\right) \\
& +\beta\left[H_{\mu}-Y B_{\mu}\right](B \cdot \partial) u^{\mu} \\
= & 0 .
\end{aligned}
$$

The entropy has to be conserved at this order. Thus, we should have

$$
\begin{aligned}
X & =-\varepsilon+s T+\mu_{\mathrm{A}} n_{\mathrm{A}}+H \cdot B \equiv p, \\
H_{\mu} & =Y B_{\mu} .
\end{aligned}
$$

As a result, we obtain the constitutive relations as

$$
\begin{aligned}
T_{(0)}^{\mu v} & =\varepsilon u^{\mu} u^{v}-p \Delta^{\mu v}-H^{\mu} B^{v}, \\
J_{A(0)}^{\mu} & =n_{\mathrm{A}} u^{\mu} .
\end{aligned}
$$

\subsection{First order}

Now let us proceed to the next order. The energy-momentum tensor, the axial current and the electric field now have the first-order contributions,

$$
T^{\mu v}=T_{(0)}^{\mu v}+T_{(1)}^{\mu v}, \quad J_{\mathrm{A}}^{\mu}=J_{\mathrm{A}(0)}^{\mu}+J_{\mathrm{A}(1)}^{\mu}, \quad E^{\mu}=E_{(1)}^{\mu} .
$$

We try to find the expressions of the first-order terms, using the second law. The equation of motions now read

$$
\begin{gathered}
u_{v} \partial_{\mu} T^{\mu v}=\partial_{\tau} \varepsilon+(\varepsilon+p) \theta+H_{v}(B \cdot \partial) u^{v}+u_{v} \partial_{\mu} T_{(1)}^{\mu v}=0, \\
\partial_{\tau} n_{\mathrm{A}}+n_{\mathrm{A}} \theta+\partial \cdot J_{\mathrm{A}(1)}=C_{\mathrm{A}} E_{(1)} \cdot B . \\
\partial_{\tau} B^{\mu}+B^{\mu} \theta-(B \cdot \partial) u^{\mu}-u^{\mu}(\partial \cdot B)-\partial_{v}\left(\varepsilon^{\mu v \alpha \beta} u_{\alpha} E_{(1) \beta}\right)=0 .
\end{gathered}
$$

The divergence of the entropy current, $S^{\mu}=s u^{\mu}+S_{(1)}^{\mu}$, reads

$$
\begin{aligned}
\partial \cdot S= & \beta\left(-u_{v} \partial_{\mu} T_{(1)}^{\mu v}\right)-\bar{\mu}_{\mathrm{A}}\left(-\partial \cdot J_{A(1)}+C_{\mathrm{A}} E_{(1)} \cdot B\right) \\
& -\beta H_{\mu} \partial_{v}\left(\varepsilon^{\mu v \alpha \beta} u_{\alpha} E_{(1) \beta}\right)+\partial \cdot S_{(1)} \\
= & T_{(1)}^{\mu v} \partial_{\mu}\left(\beta u_{v}\right)-J_{A(1)} \cdot \partial \bar{\mu}_{\mathrm{A}} \\
& +E_{(1) \beta}\left(-C_{\mathrm{A}} \bar{\mu}_{\mathrm{A}} B^{\beta}-\varepsilon^{\mu v \alpha \beta} \partial_{\mu}\left(\beta H_{v}\right) u_{\alpha}\right),
\end{aligned}
$$


where we have set

$$
S_{(1)}^{\mu}=\beta u_{v} T_{(1)}^{\mu \nu}-\bar{\mu}_{\mathrm{A}} J_{\mathrm{A}(1)}^{\mu}-\varepsilon^{\mu v \alpha \beta} \beta H_{v} u_{\alpha} E_{(1) \beta} .
$$

The positivity or the entropy production is assured if the following conditions are met,

$$
\begin{gathered}
T_{(1)}^{\mu v} \partial_{\mu}\left(\beta u_{v}\right) \geq 0, \\
-J_{A(1)} \cdot \partial \bar{\mu}_{\mathrm{A}} \geq 0, \\
E_{(1) \mu}\left[-C_{\mathrm{A}} \bar{\mu}_{\mathrm{A}} B^{\mu}+\varepsilon^{\mu v \alpha \beta} u_{v} \partial_{\alpha}\left(\beta H_{\beta}\right)\right] \geq 0 .
\end{gathered}
$$

We can satisfy Eqs. (3.25) and (3.26) by introducing the viscosities and diffusion constant as ${ }^{1}$

$$
T_{(1)}^{\mu v}=\eta \nabla^{<\mu} u^{v>}+\zeta \nabla \cdot u
$$

where the brackets $\langle\ldots\rangle$ denotes the symmetric and traceless part, and

$$
J_{\mathrm{A}(1)}^{\mu}=-D_{\mathrm{A}} \nabla^{\mu} \bar{\mu}_{\mathrm{A}},
$$

with $\nabla^{\mu} \equiv \Delta^{\mu v} \partial_{v}$. For Eq. (3.27), we should have

$$
\left[-C_{\mathrm{A}} \bar{\mu}_{\mathrm{A}} B^{\mu}+\varepsilon^{\mu v \alpha \beta} u_{v} \partial_{\alpha}\left(\beta H_{\beta}\right)\right]=X^{\mu v} E_{(1) v},
$$

where $X^{\mu v}$ is a positive semi-definite matrix. Let us introduce a unit vector along the magnetic field as

$$
b^{\mu} \equiv \frac{B^{\mu}}{\sqrt{-B^{2}}},
$$

which satisfies $b_{\mu} b^{\mu}=-1$. Let us decompose the spatial projector as

$$
\Delta^{\mu v}=-b^{\mu} b^{v}+\Delta_{\perp}^{\mu v}
$$

where $\Delta_{\perp}^{\mu v} \equiv \Delta^{\mu v}+b^{\mu} b^{v}$. The tensor $X^{\mu v}$ can be written as

$$
X^{\mu v}=-\beta\left[-\sigma_{\|} b^{\mu} b^{v}+\sigma_{\perp} \Delta_{\perp}^{\mu v}+\sigma_{\text {Hall }} \varepsilon^{\mu v \alpha \beta} u_{\alpha} b_{\beta}\right],
$$

where $\sigma_{\|}$and $\sigma_{\perp}$ are parallel and perpendicular conductivities, and $\sigma_{\text {Hall }}$ is the Hall conductivity. The Hall current does not contribute to entropy production. For simplicity, let us look at the case $\sigma_{\|}=\sigma_{\perp} \equiv \sigma$ and $\sigma_{\text {Hall }}=0$. The we obtain the expression for the first-order electric field as

$$
E_{(1)}^{\mu}=-\frac{1}{\sigma \beta} \varepsilon^{\mu v \alpha \beta} u_{v} \partial_{\alpha}\left(\beta H_{\beta}\right)+\frac{C_{\mathrm{A}} \mu_{\mathrm{A}}}{\sigma} B^{\mu}
$$

The second term on the right in Eq. (3.34) represents the chiral magnetic effect.

\footnotetext{
${ }^{1}$ The viscosities and the diffusion constant can be anisotropic in general, because of the existence of the magnetic field $[16,17,18,19,20]$
} 


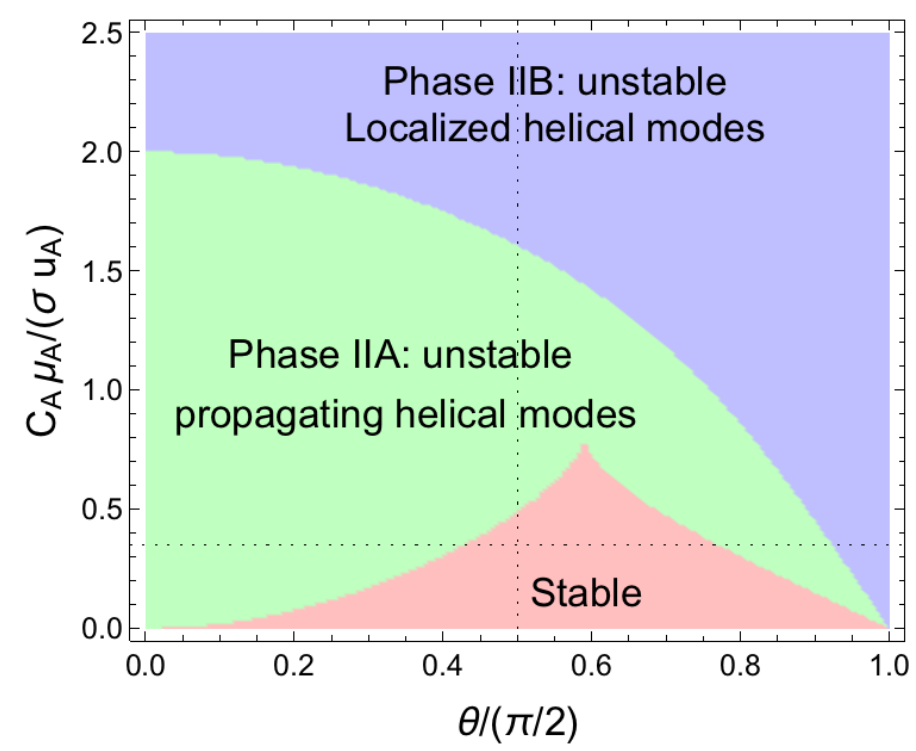

Figure 2: Behaviors of collective modes as a function of $\theta$ and $\varepsilon_{\mathrm{A}}$ for $c_{\mathrm{s}} / u_{\mathrm{A}}=0.6$.

\subsection{Collective excitations}

Let us now discuss the properties of collective modes in the chiral MHD. We consider a chiral fluid with $u_{0}^{\mu}=(1,0,0,0)$ under a homogeneous magnetic field $B_{0}^{\mu}$. The energy density $\varepsilon^{0}$, and axial charge density $n_{\mathrm{A}}^{0}$ are also homogeneous. We look at the linear fluctuations around this background.

In the case of idea MHD, the magnetic field is frozen in to the matter. The fluid and the magnetic field always move together. There is a wave called the Alfvén mode, which propagates along the magnetic field. When the wave is propagating along the magnetic field, we can obtain the dispersion relation of the Alfvén wave modified by the chiral anomaly, as

$$
\omega= \pm u_{A} k_{\|}-\frac{i}{2}\left[(\bar{\eta}+\lambda) k_{\|}^{2}-s \varepsilon_{\mathrm{A}} k_{\|}\right]
$$

where $\varepsilon_{\mathrm{A}} \equiv C_{\mathrm{A}} \mu_{\mathrm{A}} / \sigma, u_{\mathrm{A}} \equiv \sqrt{B^{2} /\left(e+p+B^{2}\right)}$ is the Alfvén velocity, $\bar{\eta} \equiv \eta /\left(e+p+\boldsymbol{B}^{2}\right)$ is a rescaled shear viscosity, and $s$ is the helicity of the wave. Helicity-dependent instability appears, due to the contribution proportional to $\varepsilon_{\mathrm{A}}$. When $\mu_{\mathrm{A}}>0$, the positive helicity modes with $k<k_{c}$ is unstable, where

$$
k_{\mathrm{c}}=\frac{C_{\mathrm{A}} \mu_{\mathrm{A}}}{1+\bar{\eta} \sigma} .
$$

The instability leads to the generation of helical magnetic fields and flow velocities.

In Fig. 2, we have summarized the nature of waves $^{2}$. There are six modes in total, which are all massless. The horizontal axis $\theta$ represents the angle between the wave vector and the magnetic field. In the red region, all the waves are stable. In the green blue regions, unstable modes appear.

\footnotetext{
${ }^{2}$ In this plot, $\eta=\zeta=D_{\mathrm{A}}=0$, which corresponds to the small $k$ limit, and a non-relativistic limit is taken where $u_{\mathrm{A}}$ is small.
} 
The instability found here is in line with the instabilities discussed for chiral plasmas[21, 22]. It would be interesting to investigate the fate of the instability in the hydrodynamic regime.

\section{Potentials of chiral MHD}

Having formulated the basis of chiral MHD, we are now in position to apply the theory to describe real-world phenomena. The chiral MHD would be an essential tool to look for the evidence of anomaly-induced transport effects in heavy-ion collisions. A hydrodynamic model including the chiral MHD can give an quantitative prediction of the observables.

Description in terms of chiral MHD is also important for early Universe before electroweak phase transition. The interplay of chiral fermions with dynamical gauge fields leads to a formation of large-scale magnetic fields like we see in the current Universe. The chiral MHD has the potential to explain the reason how the large-scale magnetic fields are developed.

It can be applied to recently discovered Dirac/Weyl semimetals, in which the quasiparticles show relativistic dispersions. Fluid-dynamic behavior of electrons in graphene is experimentally discovered recently[23]. It opens the possibility of developing new electronic devices utilizing the fluid-like and anomalous properties of the materials. The anomalous transport effects involve "chiralities. "Current semiconductor technology is based on the transport of electric charges. The chiral transport effects make it possible to use the chirality for information processing. In order to develop the methods of chirality manipulations, we have to understand the nature of 3D chiral materials, including its interaction with (polarized) photons. The chiral MHD is an ideal tool for this purpose.

\section{Summary}

We have discussed the chiral MHD, which is a low-energy effective theory to describe chiral plasmas coupled with dynamical electromagnetic fields. The chiral MHD is formulated as a kind of hydrodynamic expansion, in terms of the number of derivatives and the power of the anomaly coefficient. The second law of thermodynamic provides us with additional constraints. The CME current naturally arises in the first-order contribution to the constitutive equation of the electric field. We find an instability that leads to the generation of helical magnetic fields and flow velocities.

\section{References}

[1] D. E. Kharzeev, L. D. McLerran and H. J. Warringa, The Effects of topological charge change in heavy ion collisions: 'Event by event P and CP violation', Nucl.Phys. A803 (2008) 227-253, [0711.0950].

[2] K. Fukushima, D. E. Kharzeev and H. J. Warringa, Real-time dynamics of the Chiral Magnetic Effect, Phys.Rev.Lett. 104 (2010) 212001, [1002.2495].

[3] A. Yamamoto, Chiral magnetic effect in lattice QCD with a chiral chemical potential, Phys. Rev. Lett. 107 (2011) 031601, [1105.0385]. 
[4] G. S. Bali, F. Bruckmann, G. EndrÃúdi, Z. Fodor, S. D. Katz and A. SchÃd'fer, Local CP-violation and electric charge separation by magnetic fields from lattice QCD, JHEP 04 (2014) 129, [1401.4141].

[5] D. T. Son and N. Yamamoto, Berry Curvature, Triangle Anomalies, and the Chiral Magnetic Effect in Fermi Liquids, Phys. Rev. Lett. 109 (2012) 181602, [1203.2697].

[6] D. T. Son and N. Yamamoto, Kinetic theory with Berry curvature from quantum field theories, Phys. Rev. D87 (2013) 085016, [1210.8158].

[7] M. A. Stephanov and Y. Yin, Chiral Kinetic Theory, Phys. Rev. Lett. 109 (2012) 162001, [1207.0747].

[8] D. T. Son and P. Surowka, Hydrodynamics with Triangle Anomalies, Phys.Rev.Lett. 103 (2009) 191601, [0906.5044].

[9] Q. Li, D. E. Kharzeev, C. Zhang, Y. Huang, I. Pletikosic, A. V. Fedorov et al., Observation of the chiral magnetic effect in ZrTe5, Nature Phys. 12 (2016) 550-554, [1412 . 6543].

[10] H. K. Moffatt, The energy spectrum of knots and links, Nature 347 (1990) 367-369.

[11] R. Ricca and H. Moffatt, The helicity of a knotted vortex filament, in Topological Aspects of the Dynamics of Fluids and Plasmas, pp. 225-236. Springer, 1992.

[12] H. Moffatt and R. L. Ricca, Helicity and the calugareanu invariant, in Proceedings of the Royal Society of London A: Mathematical, Physical and Engineering Sciences, vol. 439, pp. 411-429, The Royal Society, 1992.

[13] Y. Hirono, D. E. Kharzeev and Y. Yin, Quantized chiral magnetic current from reconnections of magnetic flux, Phys. Rev. Lett. 117 (2016) 172301, [1606.09611].

[14] V. P. Kirilin and A. V. Sadofyev, Anomalous Transport and Generalized Axial Charge, 1703.02483.

[15] K. Hattori, Y. Hirono, H.-U. Yee and Y. Yin, Magnetohydrodynamics with chiral anomaly: phases of collective excitations and instabilities, 1711.08450.

[16] X.-G. Huang, M. Huang, D. H. Rischke and A. Sedrakian, Anisotropic Hydrodynamics, Bulk Viscosities and R-Modes of Strange Quark Stars with Strong Magnetic Fields, Phys. Rev. D81 (2010) 045015, [0910.3633].

[17] X.-G. Huang, A. Sedrakian and D. H. Rischke, Kubo formulae for relativistic fluids in strong magnetic fields, Annals Phys. 326 (2011) 3075-3094, [1108.0602].

[18] P. Kovtun, Thermodynamics of polarized relativistic matter, JHEP 07 (2016) 028, [160 6.01226 ].

[19] S. Grozdanov, D. M. Hofman and N. Iqbal, Generalized global symmetries and dissipative magnetohydrodynamics, 1610.07392.

[20] J. Hernandez and P. Kovtun, Relativistic magnetohydrodynamics, 1703.08757.

[21] M. Joyce and M. E. Shaposhnikov, Primordial magnetic fields, right-handed electrons, and the Abelian anomaly, Phys. Rev. Lett. 79 (1997) 1193-1196, [astro-ph/9703005].

[22] Y. Akamatsu and N. Yamamoto, Chiral Plasma Instabilities, Phys. Rev. Lett. 111 (2013) 052002, [1302.2125].

[23] D. Bandurin, I. Torre, R. K. Kumar, M. B. Shalom, A. Tomadin, A. Principi et al., Negative local resistance caused by viscous electron backflow in graphene, Science 351 (2016) 1055-1058. 